Lehrveranstaltungen zum Recht für die Soziale Arbeit sind fester Bestandteil aller Bachelor- und vieler Masterstudiengänge für Sozialarbeit und Sozialpädagogik an den Hochschulen, Fachhochschulen und Universitäten in Deutschland. Und den Fachkräften der Sozialen Arbeit und Sozialverwaltung stellen sich vielfältige Rechtsfragen in der Alltagspraxis.

\title{
Aktuelle Fachliteratur zum Rehabilitationsrecht für die Soziale Arbeit
}

von

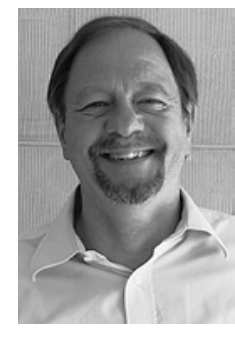

Prof. Dr. jur. Dr. phil. Reinhard Joachim Wabnitz Jg. 1952, Magister rer. publ., Ministerialdirektor a.D., Hochschule RheinMain, Fachbereich Sozialwesen

Mit Blick auf die Zielgruppen sowohl der Studierenden als auch der Fachkräfte der Sozialen Arbeit und Sozialverwaltung gibt Reinhard Wabnitz in unsere jugend etwa zweimal pro Jahr einen Überblick über aktuelle Rechtsliteratur für die Soziale Arbeit, etwa zum Allgemeinen Sozialrecht, Familienrecht, Kinder- und Jugendhilferecht, Recht der Existenzsicherungsleistungen, Rehabilitationsrecht, Sozialverwaltungsrecht oder Strafrecht/Jugendstrafrecht. Die Reihe startete in Heft 9/2014 (384ff) mit dem Schwerpunkt „Aktuelle Fachliteratur zur Einführung in das Recht für die Soziale Arbeit“.

Das Rehabilitationsrecht stellt eine sehr komplexe Materie dar. Es besteht im Wesentlichen aus Teil 1 des SGB IX (Rehabilitation und Teilhabe) sowie aus den speziellen, insoweit vorrangigen rehabilitationsrechtlichen Regelungen insbesondere im SGB II (Grundsicherung für Arbeitsu- chende), SGB III (Arbeitsförderung), SGB V (Gesetzliche Krankenversicherung), SGB VI (Gesetzliche Rentenversicherung), SGB VII (Gesetzliche Unfallversicherung), SGB VIII (Kinder- und Jugendhilfe) und SGB XII (Sozialhilfe), ergänzt zudem um die allgemeinen und verfahrensrechtlichen Regelungen in den Büchern I, IV und X des SGB. Hinzu kommen noch das Soziale Entschädigungsrecht sowie das (in sich weitgehend geschlossene) Schwerbehindertenrecht als Teil 2 des SGB IX. Auf Erläuterungswerke zu den beiden zuletzt genannten, doch sehr speziellen Rechtsgebieten werde ich hier nicht näher eingehen, verweise allerdings zum Schwerbehindertenrecht auf Gerd Cramer/Harry Fuchs/Stephan Hirsch/Hans-Günther Ritz, SGB IX - Kommentar zum Recht schwerbehinderter Menschen, 6. Aufl. 2011, 971 Seiten, Verlag Vahlen, ISBN 978-3-8006-2953-4 , € 94,- und auf Dorothee Müller-Wenner/Jürgen Winkler, SGB IX Teil 2. Besondere Regelungen zurTeilhabe schwerbehinderter Menschen (Schwerbehindertenrecht), Kommentar, 2. Aufl. 2011, 786 Seiten, Beck Verlag München, ISBN 978-3-406-61072-1, € 58,-.

Vielleicht liegt es schon an dieser Komplexität der Materie, dass das Rehabilitationsrecht im Kanon der Rechtsfächer an den Hochschulen und Fachbereichen für Soziale Arbeit leider nicht den Stellenwert eingeräumt bekommen hat, der ihm in der Praxis zukommt; von daher 
mangelt es auch an kurz gefassten Lehrbüchern für Studierende der Sozialen Arbeit. Auf der anderen Seite gibt es eine kaum noch überschaubare Vielzahl von breit und vertieft angelegten Gesamtdarstellungen für die Praxis. Diese sind zumeist auch nicht von einzelnen Autorlnnen, sondern von ganzen Herausgeber- und Autorenteams vorgelegt worden. Hinzuweisen ist etwa auf die folgenden Kommentare, deren Erscheinen allerdings bereits einige Jahre zurückliegt bzw. für welche erst für die nächste Zeit Neuauflagen angekündigt sind:

\section{Olaf Deinert/Volker Neumann (Hrsg.)}

Rehabilitation und Teilhabe

behinderter Menschen

Handbuch SGB IX, 2. Aufl. 2009, Nomos Verlag Baden-Baden, ISBN 978-3-8329-3937-3, € 98,-

Dieses Werk ist nunmehr als "Stichwortkommentar Behindertenrecht" fortgeführt worden von OlafDeinert/Felix Welti, 1. Aufl. 2014 (siehe unten)

\section{Michael Kossens/Dirk von der Heide/ Michael Maaß (Hrsg.) \\ SGB IX Rehabilitation und Teilhabe behinderter Menschen mit Behinderten- gleichstellungsgesetz}

Kommentar, 3. Aufl. 2009, 4. Aufl. vorgesehen für 2015, Beck Verlag München, voraussichtlich ca. 740 Seiten, ISBN 978-3-406-66802-9, voraussichtlich ca. $€ 79$,-

\section{Peter Mrozynski/Jürgen Jabben (Hrsg.)}

SGB IX Teil 1. Regelungen für behinderte und von Behinderung bedrohte Menschen Kommentar, 2. Aufl. 2011, BeckVerlag München, 606 Seiten, ISBN 978-3-406-61071-4, $€ 48,00$

\section{Dirk Neumann/Ronald Pahlen/}

Monika Majerski-Pahlen (Hrsg.)

Sozialgesetzbuch IX - Rehabilitation und Teilhabe behinderter Menschen: SGB IX Kommentar,12. Aufl. 2010, BeckVerlag München, 900 Seiten, ISBN 978-3-406-59161-7, $€ 98$,-
Da aus Platzgründen hier eine Rezension aller einschlägigen Werke nicht möglich ist, werden im Folgenden drei ausgewählte Bücher zum Rehabilitationsrecht im Einzelnen vorgestellt. Diese subjektive Auswahl lässt sich damit rechtfertigen, dass sie alle im Jahr 2014 neu erschienen und zugleich repräsentativ sind für die drei Gattungen: Handbuch, Stichwortkommentar und „klassischer" Kommentar. Dabei werde ich nach der Benennung der Bücher jeweils eingehen auf: HerausgeberInnen und Autorlnnen, wesentliche Inhalte des Werkes und Zielsetzungen.

\section{Handbuch}

Ernst-Wilhelm Luthe (Hrsg.)

Rehabilitationsrecht

2. Aufl. 2014, Erich Schmidt Verlag Berlin, 748 Seiten, ISBN 978-3-503-15747-1, € 98,-

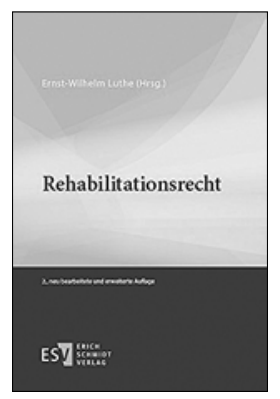

Der Herausgeber ist Professor an der Ostfalia Hochschule sowie an der Universität Oldenburg. Bearbeitet wurde das Werk außer von ihm selbst von 12 weiteren Autorlnnen aus Wissenschaft und Praxis (Johannes Falterbaum, Stephan Gutzler, Guido Kirchhoff, Wolfgang Noftz, DagmarOppermann, Ingo Palsherm, Rainer Schlegel, Wolfgang Schütte, Thomas Stähler, Peter Udsching, Felix Welti und Reinhard Wiesner).

Das Werk ist in vier Teile gegliedert. In Teil 1 (Grundlagen des Rehabilitationsrechts) werden in fünf Kapiteln A bis E auf ca. 115 Druckseiten begriffliche Fragestellungen geklärt und übergreifend die Grundprinzipien des Rehabilitationsrechts, das einschlägige Verfassungsrecht, das europäische und internationale Recht sowie die Trägerzuständigkeiten im gegliederten System des deutschen Rehabilitationsrechts behandelt, zumeist vom Herausgeber selbst und durchweg eindrucksvoll und überzeu- 
gend, etwa auch bei den Ausführungen zu Inklusion und zur UN-Behindertenrechtskonvention (S. 51ff).

Teil 2 (Rehabilitation und Teilhabe behinderter Menschen nach dem SGB IX) ist dem zuletzt genannten Gesetz gewidmet. Weitgehend entsprechend der gesetzlichen Paragrafenfolge werden in zehn Kapiteln A bis J auf über 200 Druckseiten die übergeordneten und strukturbildenden Vorschriften für das gesamte Rehabilitationsrecht, die Leistungsgrundsätze, die organisatorischen Vorschriften und Leistungen in den verschiedenen Bereichen der Rehabilitation systematisch und lehrbuchartig dargestellt.

In dem umfangreichsten Teil 3 (Einzelne Leistungsgesetze) werden in acht Kapiteln $\mathrm{A}$ bis $\mathrm{H}$ auf rund 330 Druckseiten die Kapitel und Vorschriften nach den speziellen Rehabilitationsgesetzen behandelt: nach SGB II, III, V, VI, VII, VIII und XII sowie nach dem Sozialen Entschädigungsrecht. Das Buch wird abgerundet durch einen kompakten Teil 4 (Verwaltungsverfahren und Rechtsschutz) von Peter Udsching.

Wie bereits mit der 1. Auflage 2009 wird seitens des Herausgebers auch mit der zweiten Auflage eine Gesamtdarstellung des Deutschen Rehabilitationsrechts angestrebt, auch unter Berücksichtigung seiner europa- und verfassungsrechtlichen Bezüge, seiner Strukturprinzipien sowie seiner allgemeinen Vorgaben nach dem SGB IX. Mit der 2. Auflage 2014 wird das Handbuch auf den neuesten Stand von Gesetzgebung, Rechtsprechung und Literatur gebracht - unter besonderer Berücksichtigung der Neuerungen im SGB III (Arbeitsförderung) und im SGB II (Grundsicherung). Unverändert ist das Werk um eine systematische und übersichtliche Strukturierung der Materie bemüht und in dieser Hinsicht nicht nur für juristische ExpertInnen gedacht, sondern auch für alle sonstigen in der Rehabilitationspraxis wirkenden Professionen. Herausgeber und Autorlnnen haben sich mit großem Erfolg darum bemüht, "wie in der Erstausgabe für größtmögliche Klarheit in der
Darstellung dieses komplexen Rechtsgebietes zu sorgen, ohne hierbei den Anspruch einer umfassenden Erörterung der sich stellenden Fragen aufzugeben" (siehe Vorwort zur 2. Auflage, Seite V). All diesen Zielsetzungen wird das Werk auf beeindruckende Weise gerecht.

\section{Stichwortkommentar}

\section{Olaf Deinert/Felix Welti (Hrsg.)}

StichwortKommentar Behindertenrecht. Arbeits- und Sozialrecht/Öffentliches Recht/ Zivilrecht. Alphabetische Gesamtdarstellung Nomos Verlag Baden-Baden 2014 in Kooperation mit dem Lebenshilfe-Verlag, Marburg, 1.046 Seiten, ISBN 978-3-8329-7326-1, € 98,-

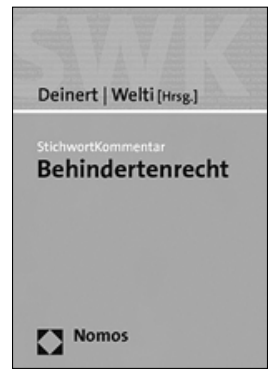

Die beiden Herausgeber sind Professoren an den Universitäten Göttingen und Kassel. Herausgeber und Verlag haben mit diesem Buch die erste Auflage eines Stichwortkommentars zum Behindertenrecht vorgelegt, wobei sie zugleich anknüpfen an das 2009 in 2. Auflage erschienene Werk "Rehabilitation und Teilhabe behinderter Menschen Handbuch SGB IX", seinerzeit herausgegeben von Olaf Deinert und Volker Neumann. Bearbeitet wurde das Werk von den Herausgebern sowie von über 20 weiteren Autorlnnen (Isabell Amann, Minou Banafsche, Claudia Beetz, Renate BieritzHarder, Uwe Boysen, Dietrich Braasch, Judith Brockmann, Wolfgang Däubler, Anna-Maria Fuerst, Christian Grube, Katharina Hilbig-Lugani, Daniel Hlava, Bernd Horstmann, Anja Jeschke, Gabriele Kuhn-Zuber, Christof Lawall, Steffen Luik, Christiane Möller, Hans-Günther Ritz, Marcus Schian, Thomas P. Stähler, Martin Theben, Peter Ulrich, Manuela Willig und Björn Winkler).

In diesem Stichwortkommentar zum Behindertenrecht (in neudeutscher Schreibweise: „StichwortKommentar") wird das Rehabilitationsrecht 
mit seinen Ausprägungen im Arbeitsrecht, Sozialrecht, sonstigen öffentlichen Recht und Zivilrecht in Form von insgesamt 162 alphabetisch geordneten Stichworten erläutert: und zwar von $A$ (Anerkennung) über B (Barrierefreiheit; Behinderung), $G$ (Gemeinsame Servicestellen), $H$ (Heilpädagogische Leistungen), $\mathrm{K}$ (Komplexleistungen), L (Leistungen an Arbeitgeber), P (Persönliches Budget), $R$ (Rehabilitation von Kindern und Jugendlichen), S (Seelische Behinderung), $\mathrm{V}$ (Verletztenrente), W (Werkstatt für behinderte Menschen) bis $Z$ (Zuständigkeit), um nur einige wenige Beispiele zu nennen. Die Ausführungen zu den einzelnen Stichworten sind je nach Erläuterungsbedarf unterschiedlich umfangreich gestaltet, von drei bis über 15 , im rechnerischen Durchschnitt von ca. sechs Druckseiten, ergänzt durch knappere oder umfangreichere Literaturhinweise. Die Qualität der Ausführungen ist nach punktueller "Durchsicht" - vorzüglich, auch bei so komplexen Themen wie etwa „Betreutes Wohnen" (von Amann, S. 196 bis 204 - auch mit detaillierten Hinweisen auf wichtige Einzelheiten in den Gesetzen der 16 Bundesländer) , "Betreuungsrecht" (von Beetz, S. 204 bis 212), ",Medizinische Rehabilitation" (von Welti, S. 598 bis 616) oder "Schule" (von Fuerst, S. 726 bis 735).

Damit beschränkt sich dieser Stichwortkommentar nicht auf das SGB IX, sondern umfasst das gesamte Behindertenrecht - auch mit Ausführungen etwa zu den Behindertengleichstellungsgesetzen, dem Allgemeinen Gleichbehandlungsgesetz und der UN-Behindertenrechtskonvention. Mit dem Stichwortkommentar Behindertenrecht soll dem Bedürfnis der Rechtspraxis Rechnung getragen werden, grundsätzliche Informationen nicht nur anhand von Paragrafen und Gesetzen, sondern auch nach Lebenssituationen und Bedarfslagen gegliedert zu erhalten. „Diese enzyklopädische Darstellung kommt zugleich neu wahrgenommenen Bedürfnissen der Leserinnen und Leser entgegen, die den schnellen Zugang zu Informationen und Analysen suchen." (Vorwort S. 5) In der Tat legt das auf zahlreiche Gesetze verteilte Behindertenrecht auch die hier gewählte Darstellungsweise nahe.

\section{„Klassischer" Kommentar}

Dirk H. Dau/Franz Josef Düwell/

Jacob Joussen (Hrsg.)

Sozialgesetzbuch IX. Rehabilitation und

Teilhabe behinderter Menschen

4. Aufl. Nomos Verlag Baden-Baden 2014, 1362 Seiten, ISBN 978-3-8487-0168-1, € 98,-

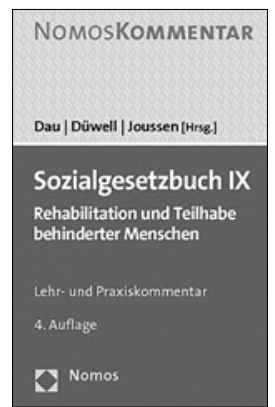

Die Herausgeber Dau und Düwell sind Vorsitzende Richter am Bundessozialgericht bzw. am Bundesarbeitsgericht a.D.; Mitherausgeber Joussen ist Professor an der Universität Bochum. Die weiteren Autoren Bertholt Deusch, Günther Hoffmann, Bernward Jacobs und Olaf Liebig sind in der Sozialverwaltung bzw. der Rechtsanwaltschaft tätig.

Dieser in der bewährten Reihe der „NomosKommentar(e)" nunmehr in 4. Auflage 2014 vorliegende "Handkommentar" ist ein "klassischer" Kommentar zu einem bestimmten Einzelgesetz, hier des SGB IX (Rehabilitation und Teilhabe behinderter Menschen). Die Kommentierungen erfolgen wie insoweit üblich in der Reihenfolge der gesetzlichen Regelungen. Nach einer instruktiven Einführung von Joussen in die Rehabilitation und Teilhabe behinderter Menschen im Sozialleistungssystem („Entwicklung eines Missstandes") sowie in die historische Entwicklung und die inhaltlichen Schwerpunkte sowie die Gliederung des Gesetzes (S. 33 bis 51) folgen auf ca. 280 Druckseiten die Erläuterungen zu den für das gesamte Rehabilitationsrecht grundlegenden Bestimmungen des Teiles 1 des SGB IX. Ausführlich kommentiert werden dessen 67 Paragrafen in den Kapiteln 1 (Allgemeine Regelungen), 2 (Ausführung von Leistungen zur Teilhabe), 3 (Gemeinsame Servicestellen), 4 (Leistungen zur medizinischen Rehabilitation), 5 (Leistungen zur Teil- 
habe am Arbeitsleben), 6 (Unterhaltssichernde und andere ergänzende Leistungen), 7 (Leistungen zur Teilhabe am Leben in der Gemeinschaft) und 8 (Sicherung und Koordinierung der Teilhabe).

Besonders ausführlich - auf über 800 Druckseiten - dargestellt wird Teil 2 des SGB IX (Besondere Regelungen zur Teilhabe schwer behinderter Menschen - Schwerbehindertenrecht) mit dessen fast 100 Paragrafen in 14 Kapiteln. Einen Schwerpunkt der Überarbeitungen bildet in dieser Auflage die Kommentierung des Wahlrechts zu den Schwerbehindertenvertretungen. An die Kommentierung des Schwerbehindertenrechts schließt sich auf ca. 55 Druckseiten eine Kommentierung der 15 Paragrafen des Gesetzes zur Gleichstellung behinderter Menschen (Behindertengleichstellungsgesetz-BGG) an. Das Werk wird ergänzt und abgerundet durch Ausführungen zu "Verfahren und Rechtsschutz" (S. 1.177 bis 1.210) und den Anhängen 1 bis 14 mit einem Komplett- oder Teilabdruck weiterer wichtiger Rechtsvorschriften (u.a. der UN-Behindertenrechtskonvention, des Bundesversorgungsgesetzes sowie auch zahlreicher Rechtsverordnungen im Bereich des Behindertenrechts).

Die Herausgeber bedauern mit den 17 Behindertenbeauftragten der Länder und des Bundes bereits im Vorwort (S. 5) die nur schleppende Umsetzung der UN-Behindertenrechtskonvention in Deutschland und die "Untätigkeit des Gesetzgebers" insoweit. Unbeschadet dessen und angesichts der bedeutsamen Rolle, die die Gerichte bei der Konkretisierung des Gesetzesrechts und bei ihrer Aufgabe der Rechtsfortbildung spielen ${ }_{\text {,r }}$ richten wir unser Hauptaugenmerk auf die Rechtsprechung. Wir bieten so der Leserschaft in Wissenschaft und Praxis eine verlässliche Orientierungshilfe" (S. 6), nachdem in den letzten drei Jahren seit Erscheinen der 3. Auflage eine Fülle höchstrichterlicher Entscheidungen ergangen ist, von denen nicht wenige mit Rechtsprechungsänderungen verbunden waren. Dabei soll der Kommentar auch in der
4. Auflage "der Praxis zu der nötigen Orientierung verhelfen und - wo erforderlich - Argumente zur Problemlösung aufzeigen“ (a.a. O.).

\section{Fazit}

Alle drei im Einzelnen vorgestellten Werke sind mit Blick auf die jeweils verfolgten Zielsetzungen sehr zu empfehlen. Man kann sie beim Studium des Rehabilitationsrechts auch gleichsam "nebeneinander legen“. Das zuletzt genannte Werk, der "klassische Kommentar", ist zweifellos besonders nützlich beim vertieften Einstieg in Spezialfragen der Praxis. Der "StichwortKommentar" vermittelt auf jeweils wenigen Druckseiten einen vorzüglichen Überblick über die 162 alphabetisch geordneten, wichtigen Themen des gesamten Behindertenrechts - bis hin zum Zivilrecht. Und das Handbuch von Luthe verbindet in gewisser Weise beides:Vermittlung von Überblicksund Detailwissen. Es zeigt systematisch die Strukturen des Behindertenrechts auf, beinhaltet umfassende Darstellungen aller wichtigen Teilgebiete und Einzelgesetze und vermittelt Einzelausführungen zu nahezu allen nur denkbaren Verästelungen des Rehabilitationsrechts.

Von daher darf ich - ohne den großen Wert auch der anderen Werke schmälern zu wollen - abschließend bemerken, dass mich das Handbuch von Luthe besonders beeindruckt. Zugleich hat Ernst-Wilhelm Luthe erneut unter Beweis gestellt, dass er einer der wirklich großen akademischen Sozial- und Verwaltungsrechtler in Deutschland ist: mit einer ungewöhnlichen Bandbreite der von inm verfassten oder herausgegebenen Publikationen etwa zum Bildungsrecht, zum Gesundheitsrecht, zum Kinder- und Jugendhilferecht, zum allgemeinen Sozialrecht und hiermit erneut zum Rehabilitationsrecht.

\footnotetext{
Prof. Dr. jur. Dr. phil. Reinhard Joachim Wabnitz Usinger Straße 104

61440 Oberursel

reinhard.wabnitz@gmx.de
} 